\title{
A Novel Method for Compact Listing of All Particular Solutions of a System of Boolean Equations
}

\author{
Ali Muhammad Ali Rushdi ${ }^{*}$ and Waleed Ahmad ${ }^{1}$ \\ ${ }^{1}$ King Abdulaziz University, P.O.Box 80204, Jeddah 21589, Saudi Arabia.
}

Authors' contributions

This work was carried out in collaboration between the two authors. Author AMAR designed the study, performed the analysis, solved the examples and wrote the manuscript. Author WA managed the literature search and drew the figures. Both authors read and approved the final manuscript.

Article Information

DOI: 10.9734/BJMCS/2017/33884

Editor(s):

(1) Radko Mesiar, Head, Department of Mathematics, Faculty of Civil Engineering, Slovak University of Technology Bratislava, Slovakia.

Reviewers:

(1) Abdullah Sonmezoglu, Bozok University, Turkey.

(2) Grienggrai Rajchakit, Maejo University, Thailand.

(3) Qingjun Kong, Tianjin Polytechnic University, China. (4) Arihant Jain, Shri Guru Sandipani Institute of Technology and Science, India. (5) Ruffin-Benoît M. Ngoie, Institut Supérieur Pédagogique, Congo. (6) K. Subramani, West Virginia University, USA. Complete Peer review History: http://www.sciencedomain.org/review-history/19556

Original Research Article

Received: $2^{\text {nd }}$ May 2017

Accepted: $7^{\text {th }}$ June 2017

Published: $15^{\text {th }}$ June 2017

\begin{abstract}
Any system of 'big' Boolean equations can be reduced to a single Boolean equation $\{g(Z)=1\}$. We propose a novel method for producing a general parametric solution for such a Boolean equation without attempting to minimize the number of parameters used, but instead using independent parameters belonging to the two-valued Boolean algebra B2 for each asserted atom that appears in the discriminants of the function $g(Z)$. We sacrifice minimality of parameters and algebraic expressions for ease, compactness and efficiency in listing all particular solutions. These solutions are given by additive formulas expressing a weighted sum of the asserted atoms of $g(Z)$, with the weight of every atom (called its contribution) having a number of alternative possible values equal to the number of appearances of the atom in the discriminants of $g(Z)$. This allows listing a huge number of particular solutions within a very small space and the possibility of constructing solutions of desirable features. The new method is demonstrated via three examples over the 'big' Boolean algebras, $B_{4}, B_{16}$, and $B_{256}$, respectively. The examples demonstrate a variety of pertinent issues such as complementation, algebra collapse, incremental solution, and handling of equations separately or jointly.
\end{abstract}

\footnotetext{
*Corresponding author: E-mail: arushdi@kau.edu.sa, arushdi@ieee.org,arushdi@yahoo.com,alirushdi@gmail.com;
} 
Keywords: 'Big' Boolean algebras; Boolean-equation solving; parametric solutions; particular solutions; contributions of atoms; Karnaugh-map like structures.

\section{Introduction}

Any system of 'big' Boolean equations can be reduced to a single Boolean equation $\{g(Z)=1\}$ or $\{\bar{g}(Z)=$ $0\}$ [1-4]. The main types of solutions of such a Boolean equation are the subsumptive general solutions, the parametric general solutions and the particular solutions. In a subsumptive general solution, each of the variables is expressed as an interval based on successive conjunctive or disjunctive eliminants of the original function [1-14]. In a parametric general solution each of the variables is expressed via arbitrary parameters which are freely chosen elements of the underlying Boolean algebra. A particular solution is an assignment from the underlying Boolean algebra to every pertinent variable that makes the Boolean equation an identity $[1,2,10,11]$. An exclusive enumeration of particular solutions is obtained from any of the two types of general solutions via an expansion tree in which a parent node might have as many children nodes as the elements in the underlying Boolean algebra [1,2,10-13]. In particular, in the conventional method for producing a general parametric solution, the number of parameters used is minimized producing compact algebraic solutions with parameters belonging to the underlying Boolean algebra. Contrarily to this convention, we propose a novel method for producing a general parametric solution that does not attempt to minimize the number of parameters used, but instead uses independent parameters belonging to the twovalued Boolean algebra B2 for each asserted atom that appears in the discriminants of the function $g(Z)$. The parametric solution obtained sacrifice minimality of parameters and algebraic expressions for ease, compactness and efficiency in listing all particular solutions. These solutions are given by additive formulas expressing a weighted sum of asserted atoms of $g(Z)$, with the weight of every atom (called its contribution) having a number of alternative possible values equal to the number of appearances of the atom in the discriminants of $g(Z)$. These alternatives are based on a set of orthonormal tags, and hence are listed in a rectangle divided into disjoint cells. This rectangle resembles a Karnaugh map, and is, in fact, a Karnaugh map, possibly with some adjacent cells combined. The representation suggested allows the possibility of listing a huge number of particular solutions within a very small space. The reason of this possibility is that an arbitrarily-selected contribution of a particular atom can be combined with any of the possible contributions of each of the other atoms. The combination via the additive (ORing) operation is simple and straightforward.

The methods presented herein could be cast in pure algebraic form $[8,11,12]$, but become much easier to visualize and comprehend when presented via the natural map of a big Boolean algebra, which (for historical reasons) is called the Variable-Entered Karnaugh Map (VEKM) [9-11,15-19]. A Boolean function of $n$ variables has $2 \mathrm{n}$ VEKM representations (depending on the choice of map and entered variables) ranging from a Conventional Karnaugh Map (CKM) which is a VEKM of $n$ map variables and 0 entered variables, to a purely-algebraic expression which is a VEKM of 0 map variables and $n$ entered variables [15]. The VEKM methods therefore include purely-algebraic methods as a special case $[11,12]$. Hence, they can always take full advantage of the results provided by the algebraic theory. Moreover, they have a better control on the minimality of pertinent function representations.

The organization of the remainder of this paper is as follows. Section 2 gives a detailed description of both the conventional and novel methods for deriving parametric solutions of 'big' Boolean equations. The section starts by pointing out the unavoidability of 'big' Boolean algebras, and then it outlines the derivation of parametric solutions before detailing ways of generating all particular solutions from them. Features of the new method are demonstrated via three examples over the 'big' Boolean algebras $B_{4}, B_{16}$, and $B_{256}$. The first example demonstrates the utility of the natural map (VEKM) of $B_{4}$ for expressing and complementing a 4-variable function and for producing the auxiliary function needed in deriving parametric solutions and subsequently in generating all particular solutions. The second example demonstrates the incremental generation of solutions of a certain equation in terms of those of another related equation. The third example lists a huge set of particular solutions (that umber slightly less than one million solutions) in a very compact space. The example also relates the individual solution of two separate equations to the joint solutions of the two equations combined as a system of equations. Section 4 concludes the paper. 


\section{Steps of the Method}

\subsection{Derivation of parametric solutions}

We seek solutions of the Boolean equation

$$
g(X, Z)=1
$$

where $g(X, Z): B_{2}^{k+n} \rightarrow B_{2}$, is a two-valued Boolean function of $k$ two-valued variables $X=\left[X_{1} X_{2} \ldots X_{k}\right]^{T}$ and $n$ two-valued variables $Z=\left[\begin{array}{llll}Z_{1} & Z_{2} & \ldots & Z_{n}\end{array}\right]^{T}$. However, we do not need a listing of binary solutions for $X$ and $Z$, but instead we want to express $Z$ in terms of $X$. This is a prominent case when the use of 'big' Boolean algebras (ones other than the two-valued algebras) is unavoidable. We view $g(X, Z)$ as $g(X ; Z)$ or simply $g(Z)$ and rewrite (1) as

$$
g(Z)=1
$$

where $g(Z): B_{2^{K}}^{n} \rightarrow B_{2^{K}}$, and $B_{2^{K}}$ is the free Boolean algebra $F B\left(X_{1}, X_{2} \ldots \ldots X_{k}\right)$ with $K=2^{k}$ atoms and $2^{K}$ elements [1,19]. Now we express $g(Z)$ by its Minterm Canonical Form (MCF) [1].

$$
g(Z) \equiv \mathrm{V}_{\mathrm{A} \in\{0,1\}^{n}} g(A) Z^{A}
$$

For $=\left[\begin{array}{lll}Z_{1} Z_{2} & \ldots & Z_{n}\end{array}\right]^{T} \in B_{2^{K}}^{n}, A=\left[\begin{array}{llll}a_{1} a_{2} & \ldots & a_{n}\end{array}\right]^{T} \in\{0,1\}^{n}$, the symbol $Z^{A}$ is defined as

$$
Z^{A}=Z_{1}^{a_{1}} Z_{2}{ }^{a_{2}} \ldots Z_{n}^{a_{n}}
$$

where $Z_{i}{ }^{a}$ takes the value $\bar{Z}_{i}$ (complemented literal) if $a_{i}=0$, and takes the value $Z_{i}$ (uncomplemented literal) if $a_{i}=1$. For $\mathrm{A} \in\{0,1\}^{n}$, the symbol $Z^{A}$ spans the minterms of $Z$, which are the $2^{n}$ elementary or primitive products

$$
\overline{Z_{1} Z_{2}} \ldots \overline{Z_{n-1} Z_{n}}, \overline{Z_{1} Z_{2}} \ldots \overline{Z_{n-1}} Z_{n}, \ldots, Z_{1} Z_{2} \ldots Z_{n-1} Z_{n}
$$

The constant values $g(A)$ in equation (2) are elements of $B_{2^{K}}$ called the discriminants of $g(Z)$ [1]. These discriminants are the entries of the natural map of $g(Z)$ which has an input domain $\{0,1\}^{n} \subseteq B_{2^{K}}^{n}$. The Boolean algebra $B_{2^{K}}=F B\left(X_{1}, X_{2} \ldots \ldots X_{k}\right)$, has generators $X_{i}(1 \leq i \leq k)$ which look like variables (In fact, they were originally our input variables before we changed their roles to generators). Therefore, we can accept the name assigned (for historical reasons) to the natural map of $g(Z)$, namely the name of the Variable-Entered Karnaugh Map (VEKM). We now observe that the minterms of $X$, which are the $2^{k}=K$ elementary or primitive products

$$
\overline{X_{1} X_{2}} \ldots \overline{X_{k-1} X_{k}}, \overline{X_{1} X_{2}} \ldots \overline{X_{k-1}} X_{k}, \ldots X_{1} X_{2} \ldots X_{k-1} X_{k}
$$

are exactly the atoms of the underlying Boolean algebra. For convenience, we call these atoms $T_{i}(0 \leq i \leq$ $(K-1)$ ), and hence $g(A)$ can be written as

$$
g(A)=\mathrm{V}_{i=0}^{K-1}\left(e_{i}(A) \wedge T_{i}\right)
$$

where we use the symbol $e_{i}(A)$ to denote an indicator of the event that atom $T_{i}$ appears in the expression of $g(A)$, i.e.,

$$
e_{i}(A)=\left\{\begin{array}{l}
1, \text { if } T_{i} \rightarrow g(A) \\
0, \text { otherwise }
\end{array}\right\}=g(A) / T_{i}
$$


where the symbol $(r / s)=(r)_{s=1}$ denotes the Boolean quotient of $r$ by $s$ [4]. Equation (7) means that $e_{i}(A)$ indicates whether atom $T_{i}$ appears in the cell $A$ of the natural map for $g(Z)$. Now, we define $n_{i}$ as the total number of actual appearances of $T_{i}$ in the expression (6) for $g(A)$, i.e.,

$$
n_{i}=\sum_{\mathrm{A} \in\{0,1\}^{n}} e_{i}(A) .
$$

The total number $N_{u}$ nconditiona 0 f unconditional particular solutions of (1a) over $B_{2^{K}}$ (as it is) is given by

$$
N_{u \text { nconditional }}=\prod_{i=0}^{K-1} n_{i} .
$$

This number is zero if some $n_{i}=0$, i.e., if an atom $T_{i}$ never makes its way to any expression $g(A)$ where $A \in\{0,1\}^{n}$ (i.e., if $T_{i}$ does not appear in any cell of the map for $g(Z)$ ). To avoid such a situation, one must insist on the consistency condition that any atom $T_{i}$ such that $n_{i}=0$ must be forbidden or nullified $[2,19]$. This means that the underlying Boolean algebra loses these atoms and hence collapses to a smaller algebra, i.e., to one of its strict sub algebras. The number of solutions over this new Boolean algebra is

$$
N_{\text {conditional }}=\prod_{\substack{i=0 \\ n_{i} \neq 0}}^{K-1} n_{i}
$$

Now we introduce a set of parameters $p_{i}\left(0 \leq i \leq(K-1), n_{i} \neq 0\right)$ to construct an orthonormal set of tags to attach to instances of appearances of the asserted atom $T_{i}$ in the discriminants $g(A)$ (i.e., in the cells $A \in\{0,1\}^{n}$ of the natural map of $g(Z)$ ). The number of parameters for atom $T_{i}$ (the length of vector $p_{i}$ ) is given by

$$
l\left(p_{i}\right)=\left\lceil\log _{2} n_{i}\right\rceil, \quad 0 \leq i \leq(K-1), n_{i} \neq 0 .
$$

Here, $\lceil x\rceil$ denotes the ceiling of the real number $x$, i.e., the smallest integer greater than or equal to $x$. The parameters $p_{i}$ can be used to generate a set of $n_{i} \leq 2^{l\left(p_{i}\right)}$ orthonormal tags $\left\{t_{1}, t_{2} \ldots t_{n_{i}}\right\}$, such that

$$
\begin{aligned}
& t_{1} \vee t_{2} \vee \ldots \vee t_{n_{i}}=1 \\
& t_{j_{1}} \wedge t_{j_{2}}=0 \quad \forall j_{1}, j_{2} \in\left\{1,2, \ldots, n_{i}\right\} .
\end{aligned}
$$

We now consider the simple case of $n_{i}=2^{l\left(p_{i}\right)}$, specifically when $n_{i}=8$ and $l\left(p_{i}\right)=3$. Fig. 1(a) demonstrates how a Karnaugh map of three variables $p_{1}, p_{2}$, and $p_{3}$ can be conveniently used to construct an element orthonormal set which consists of minterms over $\left\{p_{1}, p_{2}, p_{3}\right\}$, namely

$$
\left\{\overline{p_{1} p_{2} p_{3}}, \overline{p_{1} p_{2}} p_{3}, \overline{p_{1}} p_{2} \overline{p_{3}}, \overline{p_{1}} p_{2} p_{3}, p_{1} \overline{p_{2} p_{3}}, p_{1} \overline{p_{2}} p_{3}, p_{1} p_{2} \overline{p_{3}}, p_{1} p_{2} p_{3}\right\} \text {. }
$$

The specific value of this set is shown for each of the map cells in Fig. 1(a) in a vivid illustration of the concept of orthonormality given by (12). Now we consider the case $2^{l\left(p_{i}\right)-1}<n_{i}<2^{l\left(p_{i}\right)}$. Fig. 1(b) and 1(c) demonstrate the case $n_{i}=5$, for which $l\left(p_{i}\right)=3$. We have many possibilities for constructing an orthonormal set. Two notable examples are shown in Fig. 1(b) and 1(c) and represent, respectively the two orthonormal sets.

$$
\left\{\overline{p_{1}}, p_{1} \overline{p_{2} p_{3}}, p_{1} \overline{p_{2}} p_{3}, p_{1} p_{2} \overline{p_{3}}, p_{1} p_{2} p_{3}\right\}
$$

$$
\left\{\overline{p_{1} p_{2}}, \overline{p_{1}} p_{2}, p_{1} \overline{p_{2}}, p_{1} p_{2} \overline{p_{3}}, p_{1} p_{2} p_{3}\right\}
$$

When each appearance of an atom $T_{i}$ is tagged by a particular member of its orthonormal set of tags, an auxiliary function $G\left(Z, p_{i}\right)\left(0 \leq i \leq(K-1), n_{i} \neq 0\right)$ results. The parametric solution is now given by $[1,18-23]$. 


$$
Z_{l}=\mathrm{V}_{\left\{\mathrm{A} \in\{0,1\}^{n} \mid A_{l}=1\right\}} G\left(A, p_{i}\right) .1 \leq l \leq n,\left(0 \leq i \leq(K-1), n_{i} \neq 0\right)
$$

The total number of parameters used in (16) is given by

$$
E=\sum_{i=1}^{k} l\left(p_{i}\right)=\sum_{i=1}^{k}\left\lceil\log _{2}\left(n_{i}\right)\right]
$$

The conventional method is to select the parameter vectors from a shared pool of parameters so as to minimize the number of parameters used, which then becomes

$$
E^{\prime}=\max _{i} l\left(p_{i}\right)=\max _{i}\left[\log _{2} n_{i}\right\rceil=\left\lceil\log _{2}\left(\max _{i} n_{i}\right)\right\rceil .
$$

However, parameters used must then belong to the underlying Boolean algebra (possibly collapsed due to the consistency condition). We now propose to use independent parameters $p_{i}$ for each atom $T_{i}(0 \leq i \leq$ $K-1, n_{i} \neq 0$ ). The expressions (16) will not be as compact as they are in the conventional case, but the independent parameters $p_{i}$ now belong to the two-valued Boolean algebra $B_{2}[2,19]$, a fact that facilitates the generation of all particular solutions as we will see shortly in the next subsection.

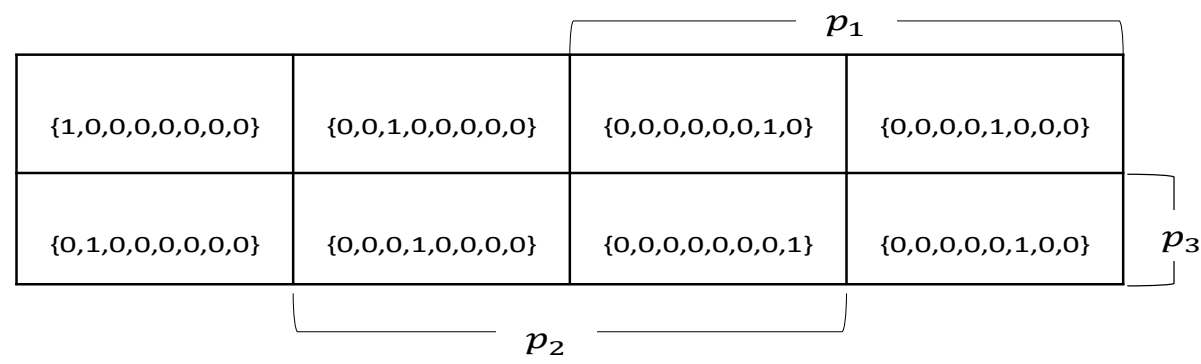

(a)

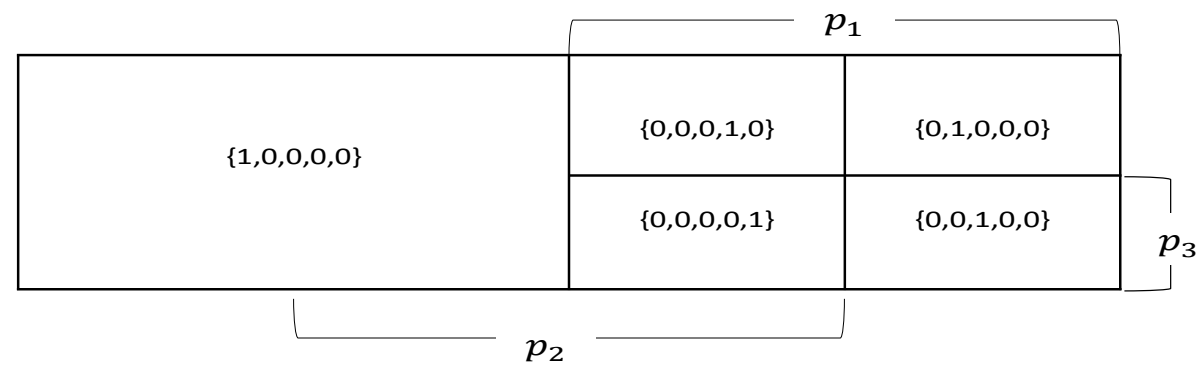

(b)

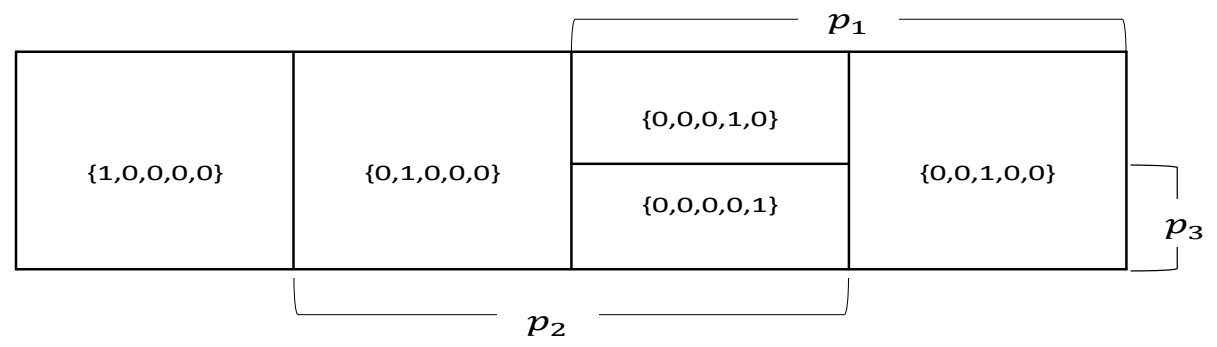

(c)

Fig. 1. Use of Karnaugh-map and Karnaugh-map like structures to construct orthonormal sets of tags 


\subsection{Listing of all particular solutions}

The parametric solutions (16) can be used to generate all particular solutions through the use of an expansion tree $[2,11,18,19]$. Generally, in the conventional method, this expansion tree is a complete tree that entails the assignment of $2^{K^{\prime}}$ values to each of $E^{\prime}$ parameters where $K^{\prime} \leq K$ is the final number of atoms of the underlying Boolean algebra (possibly after some collapse due to the consistency condition). Each parent node has $2^{K^{\prime}}$ children nodes and the tree has $E^{\prime}$ levels beyond its root. Therefore, the tree has $\left(2^{K^{\prime}}\right)^{E^{\prime}}=$ $2^{K^{\prime} E^{\prime}}$ leaves. These leaves constitute the whole set of particular solutions, possibly with repetitions. However, to avoid repetitions, we make sure, right from the first expansion level, to combine any sibling nodes that share the same solution value. With this kind of combining, the tree ceases to be a complete one, and its leaves become exactly the particular solutions, i.e., without repetitions [11].

In the method proposed herein, a complete version of the expansion tree requires the assignment of binary values $\{0,1\}$ to each of $E$ independent parameters. Since the complete binary tree has $E$ levels beyond its root, it has $2^{E}$ leaves. With merging of sibling nodes of equal solution values, the tree is no longer complete, and its leaves are just the particular solutions without repetitions. The size of the expansion tree in the proposed method is typically less than that in the conventional method since typically $E<K^{\prime} E^{\prime}$ (though $E>E^{\prime}$ ). However, the true advantage of the proposed method is that it allows us to avoid the use of an expansion tree altogether. The key of this is the observation that the parametric solution (16) can be rewritten as the weighted sum of the atoms $T_{i}$ that appear in the discriminants $g(A)$ (as expressed in (6)) of the function $g(Z)$, viz.

$$
Z=\underset{\substack{i=0 \\ n_{i} \neq 0}}{K-1}\left(\operatorname{Co}\left(T_{i}\right) \wedge T_{i}\right)
$$

where we call the vector $\operatorname{Co}\left(T_{i}\right)$ the 'contribution' of the asserted atom $T_{i}$ and call the conjunction $\left(\operatorname{Co}\left(T_{i}\right) \wedge\right.$ $T_{i}$ ) the 'total contribution' of that atom. We now note that $\operatorname{Co}\left(T_{i}\right)\left\{\right.$ or $\left.\operatorname{Co}\left(T_{i}\right) \wedge T_{i}\right\}$ has exactly $n_{i}$ possible values, which can be conveniently listed via the same Karnaugh-map-like structure used in the representation of the associated tags. Therefore, we interpret (19) as a method of conveniently listing all particular solutions as a disjunction of total contributions of asserted atoms $T_{i}$, where the total contribution is given in all its $n_{i}$ possibilities. To obtain a specific particular solution, one has simply to pick up arbitrarily one of the possibilities of the total contribution for every atom, and then add the selected total contributions together. The total number of particular solutions obtained this way agrees with that given by (10).

\subsection{Picking up a particular solution of specific features}

Equation (19) is of a paramount importance, as it provides a listing of a (possibly huge) number of all particular solutions in a compact space. As such, it allows picking up certain solutions enjoying particular desirable features simply by a quick inspection of the aforementioned listing. This point will be clarified further by way of examples in the next section.

\section{Examples}

In the following, we illustrate the method of Sec. 2 via three detailed examples. The first example applies our novel method to a 4-variable problem over $B_{4}$. The second example considers two related problems over $B_{16}$, solves the second incrementally in terms of the solution of the first, and further solves the inverse problem for one of them over $B_{256}$. The third example tackles the solutions of two equations over $B_{256}$ when taken separately or collectively.

\subsection{Example 1}

Consider the Boolean function $f\left(a ; X_{1}, X_{2}, X_{3}, X_{4}\right): B_{4}^{4} \rightarrow B_{4}$, where $B_{4}=F B(a)=\{0, a, \bar{a}, 1\}$. A solution of the equation $(f=0)$ expresses the dependent variable vector $X=\left[\begin{array}{llll}X_{1} & X_{2} & X_{3} & X_{4}\end{array}\right]^{T}$ in terms of the 
independent variable $a$ (used herein as a generator of $B_{4}$ ). Suppose $f$ is given by the product-of-sums (pos) expression

$$
f=\left(a \vee X_{2}\right)\left(a \vee X_{3}\right)\left(a \vee X_{4}\right)\left(\bar{X}_{1} \vee \bar{X}_{2}\right) .
$$

We construct the natural map (VEKM) for $f$ by noting that $\mathrm{f}$ is given by [13]

$$
f=\left(0 \vee \mathrm{Co}^{\prime}(0)\right)\left(a \vee \mathrm{Co}^{\prime}(a)\right),
$$

where $\mathrm{Co}^{\circ}(0)$ and $\mathrm{Co}^{\circ}(a)$ are called the dual or conjunctive contributions of alterms 0 and $a$, respectively, and are presented by the Conventional Karnaugh Maps (CKMs) in Fig. 2(a) and 2(b). These two figures are used to produce a natural map for $f$ in Fig. 2(c). The entries of this map are then complemented in Fig. 2(d) to obtain the inverse $g=\bar{f}$ of $f$, so that our problem becomes to solve $\{g=1\}$. Since the entry 1 stands for $(a \vee \bar{a})$, Fig. 2(d) shows that the two atoms $a$ and $\bar{a}$ of $B_{4}$ appear 4 and 15 times, respectively, in the natural map for $g$. This means that the consistency condition for the Boolean equation $\{g=1\}$ is the identity $\{0=$ $0\}$ and the number of particular solutions is $4 * 15=60$. The instances of appearances of $a$ needs 15 orthonormal tags which are defined in terms of four parameters $p_{1}, p_{2}, p_{3}$, and $p_{4}$ as shown in Fig. 3(a). Likewise, Fig. 3(a) uses two additional parameters $p_{5}$ and $p_{6}$ to define 4 orthonormal tags to be associated with the instances of appearances of atom $\bar{a}$. Fig. 3(b) shows the auxiliary function $G(a, X, p)$ for this example. The parametric solution for $\{f=0\}$ or $\{g=1\}$ is given by

$$
\left[\begin{array}{l}
X_{1} \\
X_{2} \\
X_{3} \\
X_{4}
\end{array}\right]=\bar{a}\left[\begin{array}{c}
p_{1} \\
p_{2} \\
p_{3} \\
p_{4}\left(p_{1} \vee \bar{p}_{2} \vee \bar{p}_{3}\right.
\end{array}\right] \vee a\left[\begin{array}{c}
1 \\
1 \\
p_{5} \\
p_{6}
\end{array}\right] .
$$

The parametric solution (22) leads to the compact listing of all 60 particular solutions as follows:

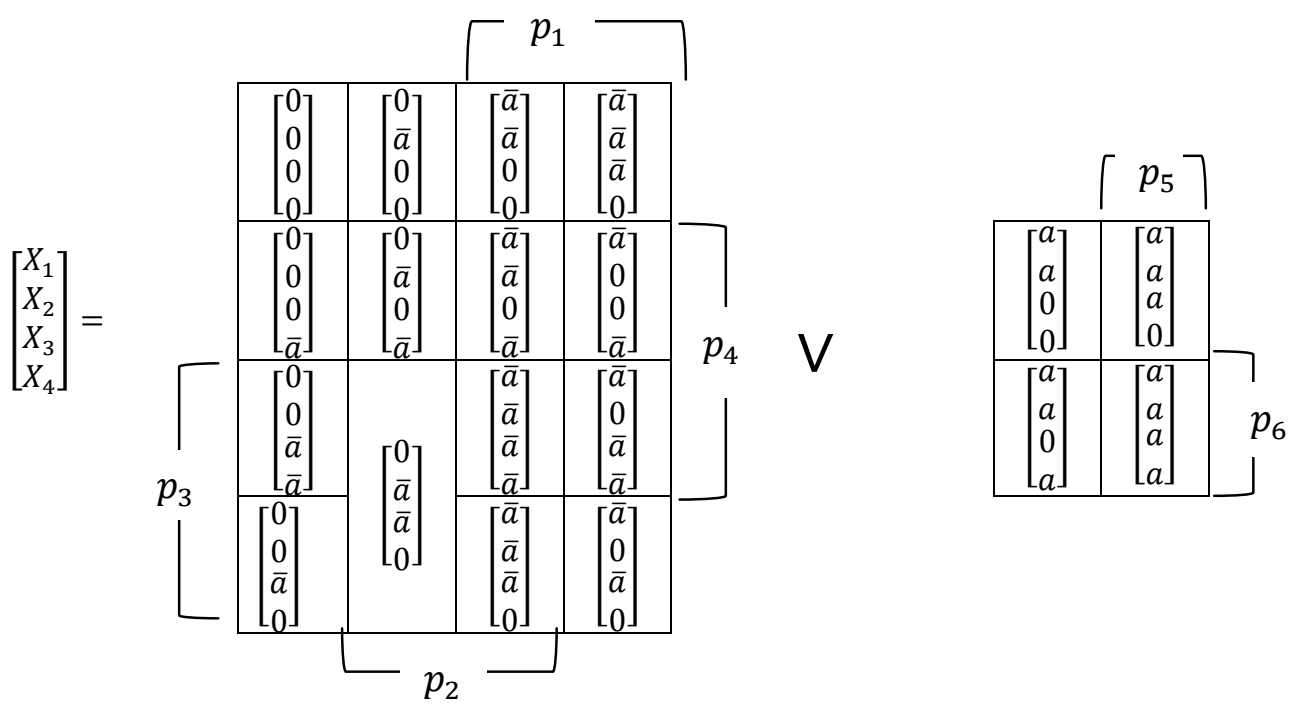

Equation (23) shows that there are 15 possibilities of total contributions $\left(\bar{a} \operatorname{Co}^{\prime}(\bar{a})\right)$ of $\bar{a}$, exhausting all 16 combinations of 4 tuples belonging each to either 0 or $\bar{a}$, with the sole exception of $\left[\begin{array}{llll}0 & \bar{a} & \bar{a} & \bar{a}\end{array}\right]^{T}$. There are also 4 possibilities of total contribution $(a \mathrm{Co}(a))$ of $a$, which are one quarter of the 16 combinations of the 4 tuples belonging each to either 0 or $a$. A particular solution is obtained by ORing an arbitrarily selected possibility of $(\bar{a} \operatorname{Co}(\bar{a}))$ with an arbitrarily selected possibility of $(a \operatorname{Co}(a))$. For example, one might implement the ORing operation of $\left[\begin{array}{llll}\bar{a} & \bar{a} & \bar{a} & \bar{a}\end{array}\right]^{T}$ with $\left[\begin{array}{llll}a & a & a & a\end{array}\right]^{T}$, to produce the solution $\left[\begin{array}{llll}1 & 1 & 1 & 1\end{array}\right]^{T}$, which can be immediately verified to produce a value of 0 for $f$ in (20). 


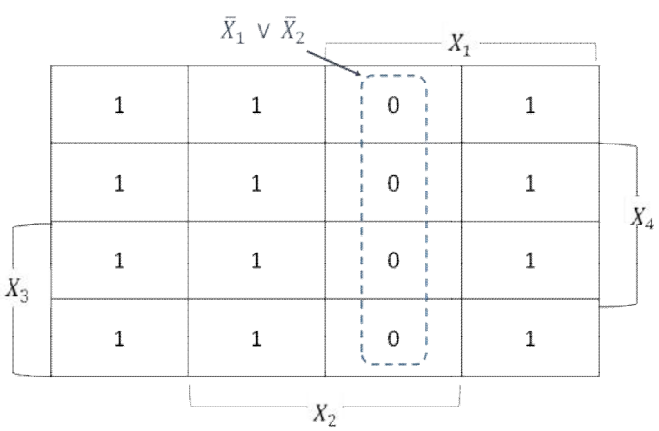

(a) $\operatorname{Co}^{\prime}(0)$

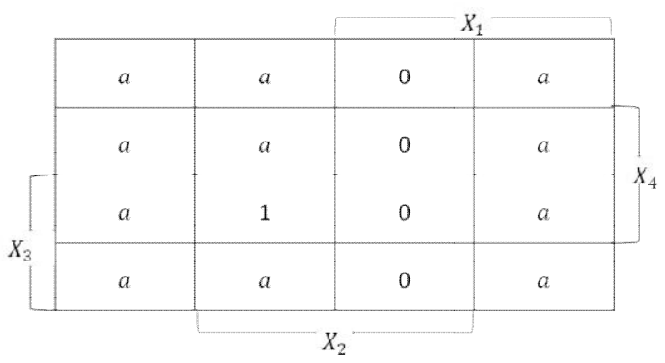

(c) $f=\left(0 \vee \mathrm{Co}^{\prime}(0)\right)\left(a \vee \mathrm{Co}^{\prime}(a)\right)$

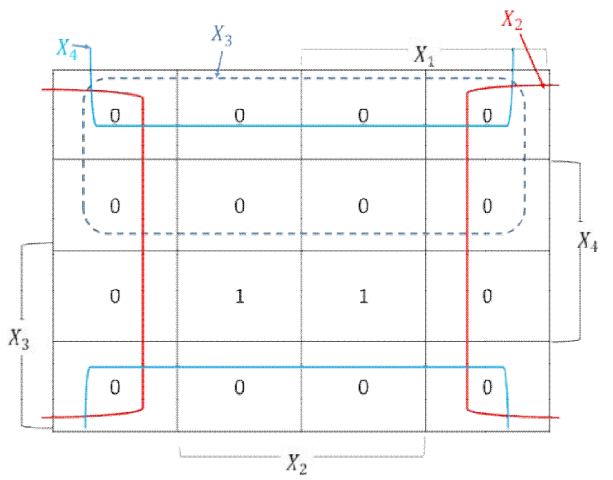

(b) $\mathrm{Co}^{\prime}(a)$

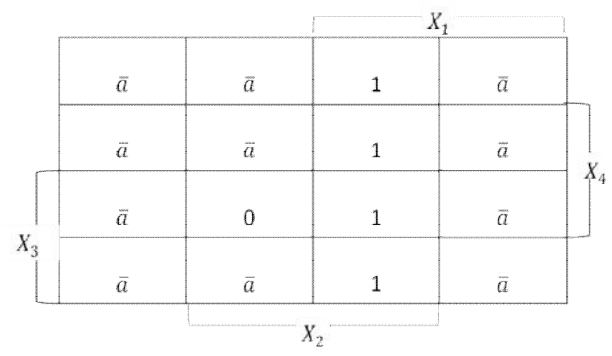

(d) $g=\bar{f}$

Fig. 2. Construction of the function $f$ in Example 1 in terms of the conjunctive contributions Co'(a) and $\mathrm{Co}^{`}(0)$, and derivation of its inverse $\mathrm{g}$ via cellwise complementation

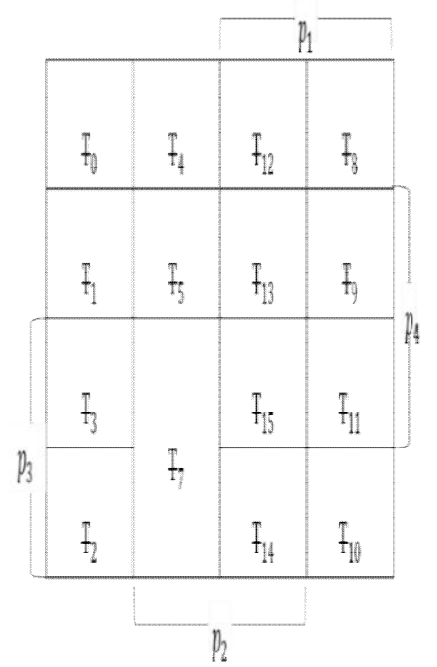

(a)
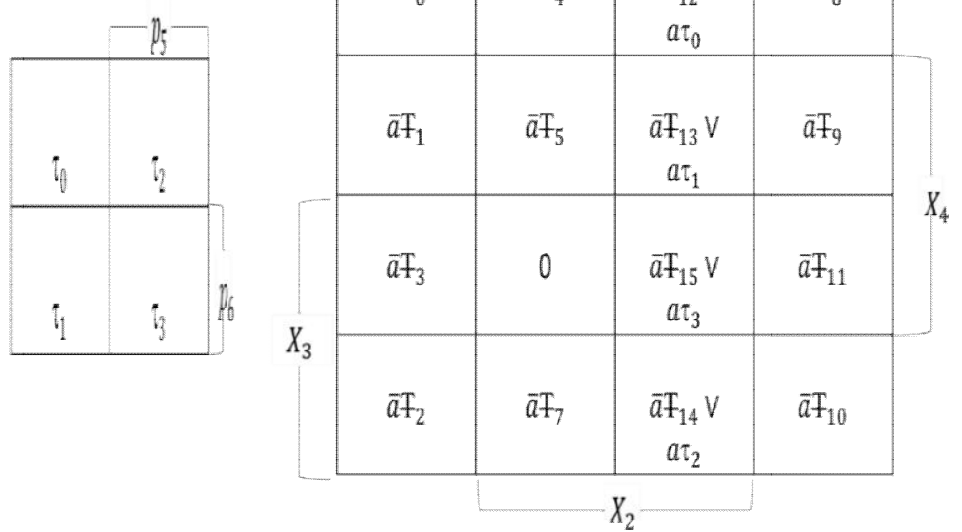

(b) $G(\boldsymbol{a}, X, p)$

Fig. 3. Definition of sets of orthonormal tags for atoms $a$ and $\bar{a}$ in Example 1. Here, for example, $T_{7}$ stands for $\bar{p}_{1} p_{2} p_{3}, T_{10}$ depicts $p_{1} \bar{p}_{2} p_{3} \bar{p}_{4}$, while $\tau_{2}$ equals $p_{5} \bar{p}_{6}$. This definition results in the auxiliary function in (b) 


\subsection{Example 2}

This example has two related versions that are very popular in the literature of Boolean equations. The first version was solved by Brown [2] and Rushdi and Amashah [18], while the second version was solved (repeatedly) by Brown [1,2] and then by Rushdi [9, 10], Rudeanu [8], and Rushdi and Amashah [19]. The first version deals with the solution of $g_{1}\left(X_{1}, X_{2}, X_{3}\right)=1$, where

$$
g_{1}\left(Y_{1}, Y_{2} ; X_{1}, X_{2}, X_{3}\right)=\bar{Y}_{1} \bar{Y}_{2}\left(X_{1} X_{2} \vee \bar{X}_{1} \bar{X}_{2} X_{3}\right) \vee Y_{1} Y_{2}\left(\bar{X}_{1} X_{2} \vee \bar{X}_{1} \bar{X}_{3}\right) \vee Y_{1} \bar{Y}_{2}\left(X_{1} \bar{X}_{2} \vee \bar{X}_{2} \bar{X}_{3}\right),
$$

is a Boolean function $g=B_{16}^{3} \rightarrow B_{16}$, where $B_{16}=F B\left(Y_{1}, Y_{2}\right)$ is a Boolean carrier of 16 elements constituting the switching functions of two variables $Y_{1}$ and $Y_{2}$. Though our equation is formulated on a big Boolean algebra, it could be thought of as one of expressing three dependent variables $X_{1}, X_{2}$ and $X_{3}$ in terms of two independent switching variables $Y_{1}$ and $Y_{2}$. Fig. 4(a) represents the natural map (VEKM) for $g_{1}$. The three atoms $\bar{Y}_{1} \bar{Y}_{2}, Y_{1} Y_{2}$, and $Y_{1} \bar{Y}_{2}$ make their appearances 3, 3, and 4 times in the 8 cells of the map of Fig. 4(a), while atom $\bar{Y}_{1} Y_{2}$ is never entered in that map. Therefore, the number of particular solutions of the equation $g_{1}=1$, is $3 * 3 * 4=36$, and the consistency condition is $\bar{Y}_{1} Y_{2}=0$. The three asserted atoms demand the use of the three sets of parameters $\left\{p_{1}, p_{2}\right\},\left\{p_{3}, p_{4}\right\}$, and $\left\{p_{5}, p_{6}\right\}$, so as to produce the three orthonormal sets of tags $\left\{p_{1}, \bar{p}_{1} p_{2}, \bar{p}_{1} \bar{p}_{2}\right\},\left\{p_{3}, \bar{p}_{3} p_{4}, \bar{p}_{3} \bar{p}_{4}\right\}$, and $\left\{p_{5} p_{6}, p_{5} \bar{p}_{6}, \bar{p}_{5} p_{6}, \bar{p}_{5} \bar{p}_{6}\right\}$, of cardinalities 3,3 , and 4 respectively. The various tags are associated with the respective atoms to construct the auxiliary function $G_{1}\left(X_{1}, X_{2}, X_{3} ; p_{1}, p_{2}, p_{3}, p_{4}, p_{5}, p_{6}\right)$ shown in Fig. 4(b). The set of parametric solutions is given by

$$
\left[\begin{array}{l}
X_{1} \\
X_{2} \\
X_{3}
\end{array}\right]=\bar{Y}_{1} \bar{Y}_{2}\left[\begin{array}{c}
p_{1} \vee \bar{p}_{1} p_{2} \\
p_{1} \vee \bar{p}_{1} p_{2} \\
p_{1} \vee \bar{p}_{1} \bar{p}_{2}
\end{array}\right] \vee Y_{1} Y_{2}\left[\begin{array}{c}
0 \\
p_{3} \vee \bar{p}_{3} p_{4} \\
p_{3}
\end{array}\right] \vee Y_{1} \bar{Y}_{2}\left[\begin{array}{c}
p_{5} p_{6} \vee \bar{p}_{5} p_{6} \\
0 \\
p_{5} p_{6} \vee p_{5} \bar{p}_{6}
\end{array}\right] \vee\left[\begin{array}{c}
d\left(\bar{Y}_{1} Y_{2}\right) \\
d\left(\bar{Y}_{1} Y_{2}\right) \\
d\left(\bar{Y}_{1} Y_{2}\right)
\end{array}\right]
$$

and could be translated into a form listing all 36 solutions, namely

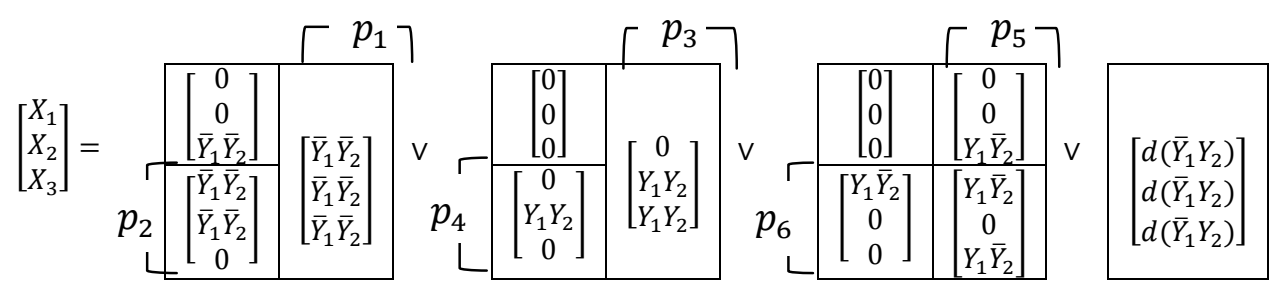

Now, we consider the second version of our current problem which is $\left\{g_{2}=1\right\}$, where $g_{2}$ is specified by Fig. 4 of Rushdi and Amashah [19], reproduced here, for convenience, as Fig. 5. A quick visual comparison of the natural maps $g_{1}$ (Fig. 4(a)) and $g_{2}$ (Fig. 5) shows that the two maps are identical except for an extra entry $Y_{1} \bar{Y}_{2}$ in the $\bar{X}_{3} X_{2} X_{1}$ cell of the map of $g_{2}$, i.e., $g_{2}$ is related to $g_{1}$ via the relation

$$
g_{2}=g_{1} \vee Y_{1} \bar{Y}_{2} \bar{X}_{3} X_{2} X_{1}
$$

The atoms $\bar{Y}_{1} \bar{Y}_{2}, Y_{1} Y_{2}, Y_{1} \bar{Y}_{2}$ and $\bar{Y}_{1} Y_{2}$ now appear 3, 3, 5, and 0 times respectively in the cells of the map in Fig. 5. Hence the equation $\left\{g_{2}=1\right\}$ again has the consistency condition $\left\{\bar{Y}_{1} Y_{2}=0\right\}$, but its number of particular solutions is now $3 * 3 * 5=45$. Note that the two functions $g_{1}$ and $g_{2}$ match with the single exception that the latter function has an extra instance of appearance of atom $Y_{1} \bar{Y}_{2}$ in the natural map of the function. Hence, the set of solutions of $\left\{g_{2}=1\right\}$ is a strict superset for the set of solutions of $\left\{g_{1}=1\right\}$. The remaining 9 solutions of $\left\{g_{2}=1\right\}$ are the solutions of the equation $\left\{g_{3}=1\right\}$ where $g_{3}$ is specified by the natural map in Fig. 6(a). That map has a single instance of appearance of the atom $Y_{1} \bar{Y}_{2}$ in the cell $\bar{X}_{3} X_{2} X_{1}$, and the same appearances of the atoms $\bar{Y}_{1} \bar{Y}_{2}$ and $Y_{1} Y_{2}$ as in either of Figs. 4(a) or 5. We now proceed to solve $\left\{g_{2}=1\right\}$ incrementally by observing that the 36 solutions of $\left\{g_{1}=1\right\}$ in (26) are also solutions of $\left\{g_{2}=1\right\}$, and that the remaining 9 solutions of $\left\{g_{2}=1\right\}$ are simply the solutions of $\left\{g_{3}=1\right\}$. To solve 
$\left\{g_{3}=1\right\}$, we use an orthonormal tag of (1) for the sole appearance of $Y_{1} \bar{Y}_{2}$ and use the same sets of parameters and tags previously used in Fig. 4(b) to obtain the auxiliary function $G_{1}\left(X_{1}, X_{2}, X_{3} ; p_{1}, p_{2}, p_{3}, p_{4}\right)$. The parametric solutions of $\left\{g_{3}=1\right\}$.are now given by

$$
\left[\begin{array}{l}
X_{1} \\
X_{2} \\
X_{3}
\end{array}\right]=\bar{Y}_{1} \bar{Y}_{2}\left[\begin{array}{c}
p_{1} \vee \bar{p}_{1} p_{2} \\
p_{1} \vee \bar{p}_{1} p_{2} \\
p_{1} \vee \bar{p}_{1} \bar{p}_{2}
\end{array}\right] \vee Y_{1} Y_{2}\left[\begin{array}{c}
0 \\
p_{3} \vee \bar{p}_{3} p_{4} \\
p_{3}
\end{array}\right] \vee Y_{1} \bar{Y}_{2}\left[\begin{array}{l}
1 \\
1 \\
0
\end{array}\right] \vee\left[\begin{array}{l}
d\left(\bar{Y}_{1} Y_{2}\right) \\
d\left(\bar{Y}_{1} Y_{2}\right) \\
d\left(\bar{Y}_{1} Y_{2}\right)
\end{array}\right]
$$

Note that the Eq. (28) can be obtained from (5) by simply changing the contribution of atom $Y_{1} \bar{Y}_{2}$ (to represent its new single instance), while keeping all other terms intact. Equation (28) produces the following set of 9 particular solutions for $\left\{g_{3}=1\right\}$.

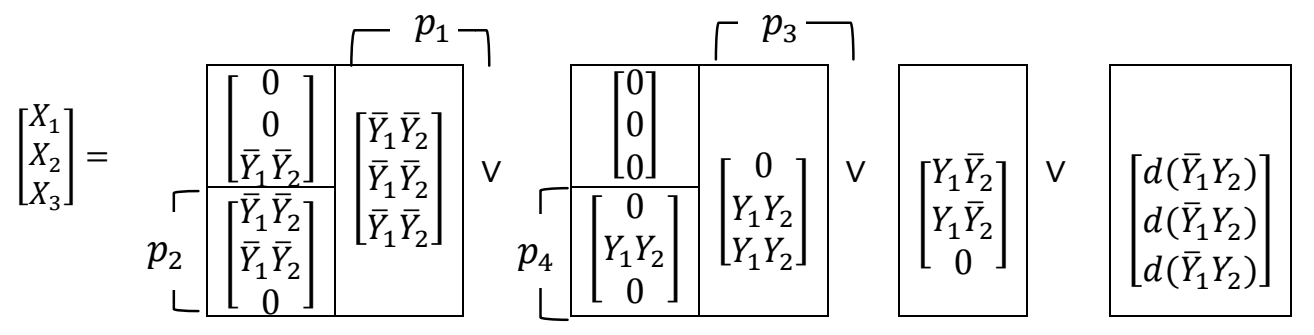

The union of the set of 36 particular solutions in (25) and the set of 9 particular solutions in (29) constitutes the set of 45 particular solutions of $\left\{g_{2}=1\right\}$. This is in agreement with earlier listings obtained via subsumptive solutions in [10] or via conventional parametric solutions in [19].

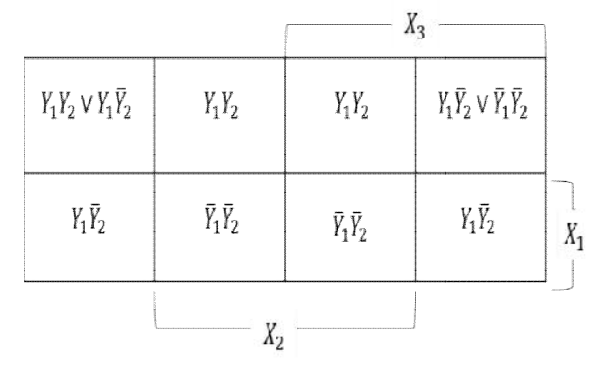

(a) $g_{3}\left(X_{3}, X_{2}, X_{1}\right)$

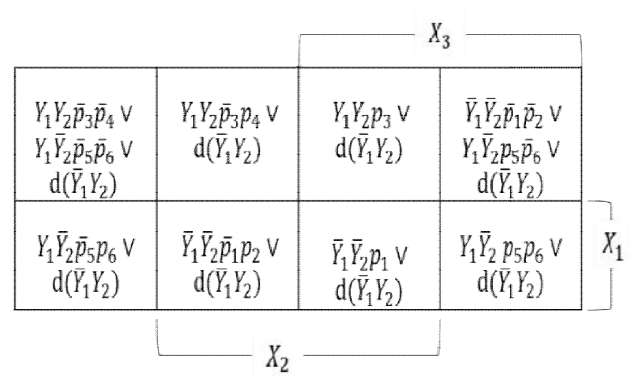

(b) $G_{3}\left(X_{3}, X_{2}, X_{1} ; p_{1}, p_{2}, p_{3}, p_{4}, p_{5}, p_{6}\right)$

Fig. 4. Natural maps for the function $g_{1}$ of Example 2 and its associated auxiliary function $G_{1}$

\begin{tabular}{|c|c|c|c|}
\cline { 3 - 4 } \multicolumn{2}{c|}{} & \multicolumn{2}{c|}{$X_{3}$} \\
\hline$Y_{1} Y_{2} \vee Y_{1} \bar{Y}_{2}$ & $Y_{1} Y_{2}$ & $Y_{1} Y_{2}$ & $Y_{1} \bar{Y}_{2} \vee \bar{Y}_{1} \bar{Y}_{2}$ \\
\hline$Y_{1} \bar{Y}_{2}$ & $Y_{1} \bar{Y}_{2} \vee \bar{Y}_{1} \bar{Y}_{2}$ & $\bar{Y}_{1} \bar{Y}_{2}$ & $Y_{1} \bar{Y}_{2}$ \\
\hline & $X_{2}$ & $X_{1}$ \\
\hline
\end{tabular}

$g_{2}(X)$

Fig. 5. The natural map for the function $g_{2}$ in Example 2 


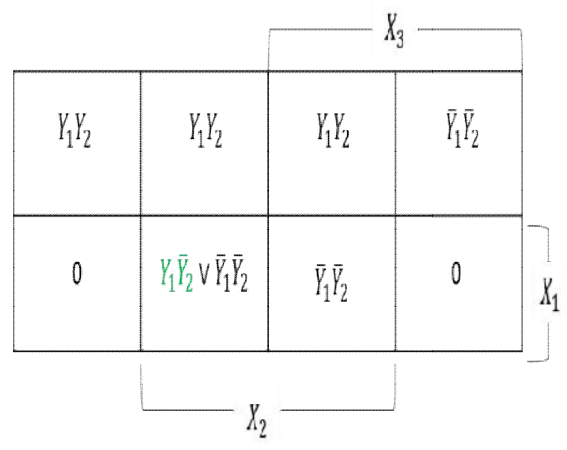

(a) $g_{3}\left(X_{3}, X_{2}, X_{1}\right)$

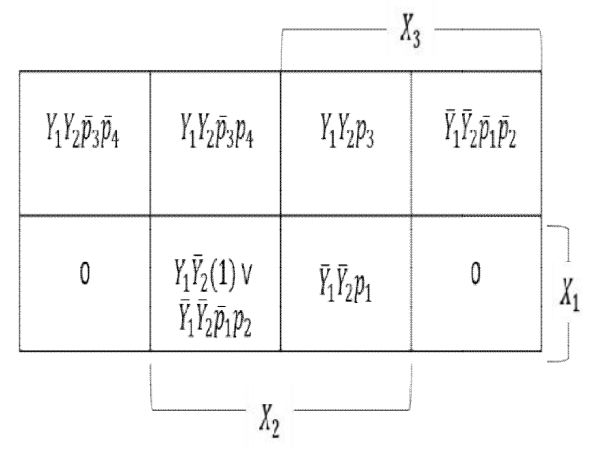

(b) $G_{3}\left(X_{3}, X_{2}, X_{1} ; p_{1}, p_{2}, p_{3}, p_{4}\right)$

Fig. 6. Natural maps for the function $g_{3}$ and its associated auxiliary function $g_{3}$ in Example 2

Now, we consider the inverse problem of solving $\left\{g_{1}=1\right\}$ to express $\boldsymbol{Y}$ in terms of $\boldsymbol{X}$. We view $g_{1}$ as $g_{1}=B_{256}^{2} \rightarrow B_{256}$, where $B_{256}=F B\left(X_{1}, X_{2}, X_{3}\right)$, and represent $g_{1}$ by the natural map in Fig. 7(a). The atoms of $B_{256}$ are $T_{0}=\overline{X_{1} X_{2} X_{3}}, T_{1}=\overline{X_{1} X_{2}} X_{3}, T_{2}=\overline{X_{1}} X_{2} \overline{X_{3}}, \quad T_{3}=\overline{X_{1}} X_{2} X_{3}, \quad T_{4}=X_{1} \overline{X_{2} X_{3}}, \quad T_{5}=$ $X_{1} \bar{X}_{2} X_{3}, T_{6}=X_{1} X_{2} \overline{X_{3}}$, and $T_{7}=X_{1} X_{2} X_{3}$. Their appearances as entries in the natural map of $g_{1}$ in Fig. 7(a) are $2,2,1,1,1,1,2$, and 1 respectively. Hence, the consistency condition is the identity $\{0=0\}$ and the number of particular solutions is $23=8$. The atoms $T_{0}, T_{1}$, and $T_{6}$ need the two element sets of tags $\left(\overline{p_{0}}, p_{0}\right)$, $\left(\overline{p_{1}}, p_{1}\right)$ and $\left(\overline{p_{6}}, p_{6}\right)$, while each of the remaining atoms have a single-element set of tag (1), each. The auxiliary function $G_{1}$ is shown in Fig. 7(b), with the parametric solutions given by

$$
\left[\begin{array}{l}
Y_{1} \\
Y_{2}
\end{array}\right]=\left[\begin{array}{c}
T_{0} \vee T_{2} \vee T_{3} \vee T_{4} \vee T_{5} \vee T_{1} p_{1} \vee T_{6} p_{6} \\
T_{0} p_{0} \vee T_{2} \vee T_{3}
\end{array}\right]
$$

and the set of eight particular solutions is listed by
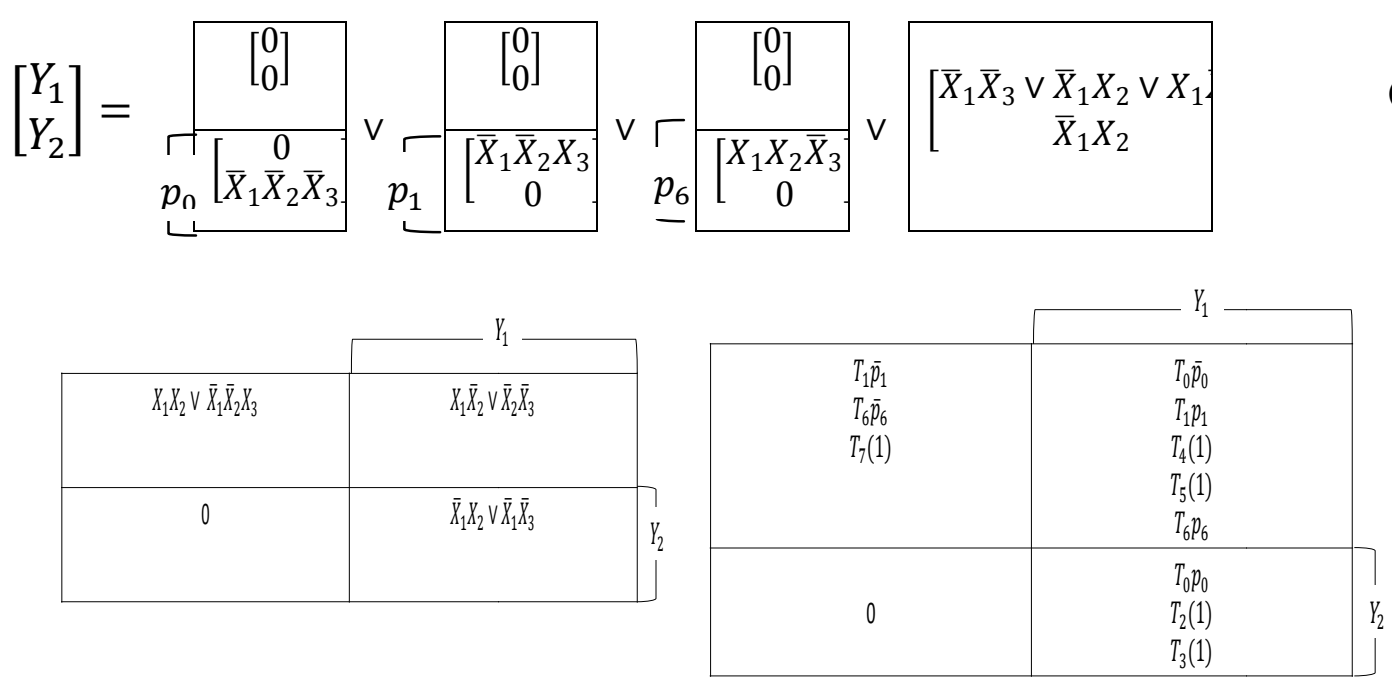

(a) $g_{1}\left(Y_{1}, Y_{2}\right)$

(b) $G_{1}\left(Y_{1}, Y_{2} ; p_{0}, p_{1}, p_{6}\right)$

Fig. 7. The natural map for the function $g_{1}$ of Example 2, now viewed as a function of $Y_{1}$ and $Y_{2}$ in terms of $X_{1}, X_{2}$, and $X_{3}$, together with the associated auxiliary function $G_{1}$ 


\subsection{Example 3}

Suppose we want to solve the system of Boolean equations

$$
\begin{aligned}
& X \vee Y \vee Z=a \vee b \vee c, \\
& \bar{X} \vee \bar{Y} \vee \bar{Z}=\bar{a} \vee \bar{b} \vee \bar{c} .
\end{aligned}
$$

for the dependent two-valued Boolean variables $X, Y$, and $Z$ in terms of the independent two-valued Boolean variables $a, b$, and $c$. An obvious solution is one that assigns to the ordered set $\{X, Y, Z\}$ the value $\{a, b, c\}$ or any of its 6 permutations. We will find shortly that other solutions exist and that the number of solutions is a huge one, indeed. We now treat the independent variables as generators of the free Boolean algebra $B_{256}=F B(a, b, c)$, which has 256 elements that represent the two-valued Boolean functions of the 3 variables $a, b$, and $c$. Clearly, this Boolean algebra has 8 atoms, viz., $T_{0}=\bar{a} \bar{b} \bar{c}, T_{1}=\bar{a} \bar{b} c, T_{2}=\bar{a} b \bar{c}$, $T_{3}=\bar{a} b c, T_{4}=a \bar{b} \bar{c}, T_{5}=a \bar{b} c, T_{6}=a b \bar{c}$, and $T_{7}=a b c$. The above system of equations reduces to a single equation of the form

$$
g_{t}(X, Y, Z)=((X \vee Y \vee Z) \odot(a \vee b \vee c)) \wedge((\bar{X} \vee \bar{Y} \vee \bar{Z}) \odot(\bar{a} \vee \bar{b} \vee \bar{c}))=1
$$

where $g_{t}(X, Y, Z): B_{256}^{3} \rightarrow B_{256}$. We represent $g_{t}(X, Y, Z)$ by its natural map in Fig. 8 . The discriminants of $g_{t}(X, Y, Z)$ entered in the map cells of Fig. 8 are obtained by noting that

$$
\begin{aligned}
& 0 \odot(a \vee b \vee c)=\overline{(a \vee b \vee c)}=\bar{a} \bar{b} \bar{c}, \text { for } X=Y=Z=0 \\
& 1 \odot(a \vee b \vee c)=(a \vee b \vee c), \text { otherwise. }
\end{aligned}
$$

Fig. 9 is a replication of Fig. 8 with map entries given as minterm expansions, i.e., as disjunctions of the atoms of the underlying Boolean algebra. We note immediately that no atom failed to appear in Fig. 9, thereby resulting in the consistency condition becoming an identity $(0=0)$ and leaving the underlying Boolean algebra intact. Each of the two atoms $T_{0}=\bar{a} \bar{b} \bar{c}$ and $T_{7}=a b c$ appears once, while each of the six atoms $T_{1}=\bar{a} \bar{b} c, T_{2}=\bar{a} b \bar{c}, T_{3}=\bar{a} b c, T_{4}=a \bar{b} \bar{c}, T_{5}=a \bar{b} c$, and $T_{6}=a b \bar{c}$ appears six times. The total number of particular solutions is given (according to (9) or (10)) by

$$
N_{t}=(1)^{2} *(6)^{6}=46656
$$

It is now our task to display this large number of solutions economically. First, we construct the auxiliary function $G\left(X, Y, Z ; p_{1}, p_{2}, p_{3}, p_{4}, p_{5}, p_{6}\right)$, where each of the six vectors of parameters $p_{\mathrm{i}}=$ $\left[\begin{array}{lll}p_{\mathrm{i} 1} & p_{\mathrm{i} 2} & p_{\mathrm{i} 3}\end{array}\right]^{T}(1 \leq i \leq 6)$ is used to produce an orthonormal set of six tags

$$
\left\{p_{i 1} \overline{p_{l 2}}, p_{i 1} p_{i 2}, \overline{p_{l 1}} p_{i 2} p_{i 3}, \overline{p_{l 1}} p_{i 2} \overline{p_{l 3}}, \overline{p_{l 1} p_{l 2}} p_{i 3}, \overline{p_{l 1} p_{l 2} p_{l 3}}\right\}
$$

The solution in vector form is

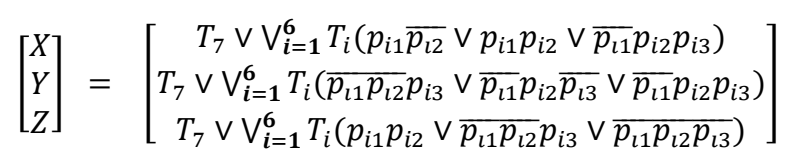

The following equation (38) displays all 46656 particular solutions of the system (25) as a disjunction of possible contributions of atoms $T_{i}(1 \leq i \leq 7)$. 


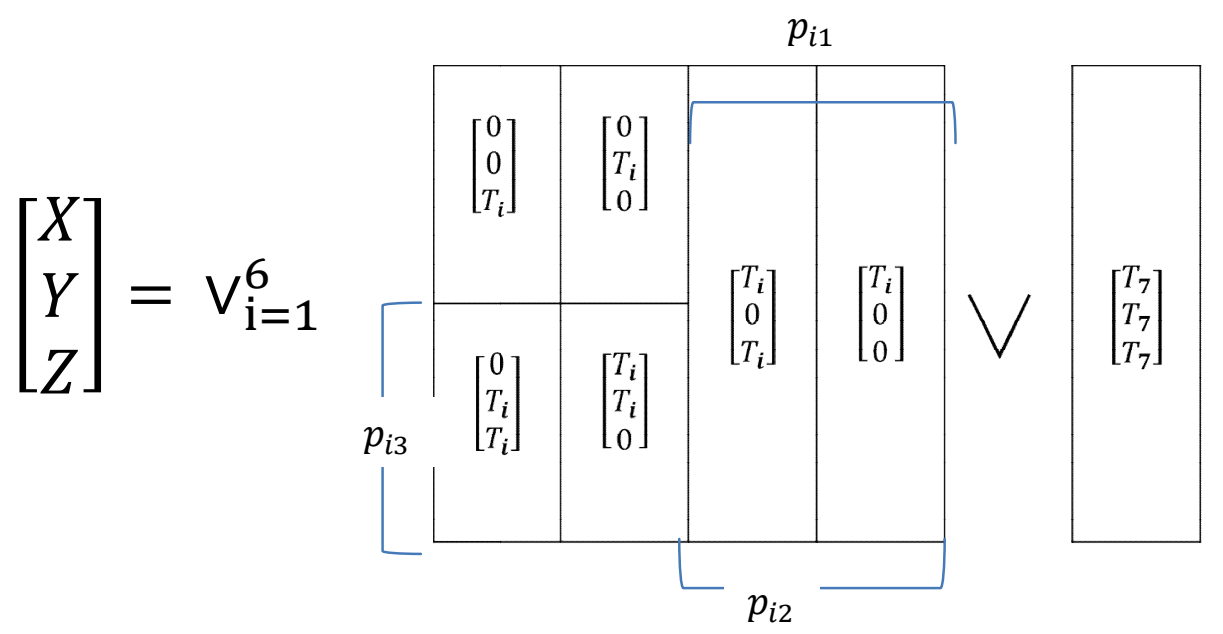

Table 1 demonstrates a few samples of the particular solutions that can be obtained via (38). The first of these is the obvious solution.

$$
\left[\begin{array}{l}
X \\
Y \\
Z
\end{array}\right]=\left[\begin{array}{l}
a \\
b \\
c
\end{array}\right] .
$$

Each of the solutions in Table 1 can be easily shown to satisfy each of the separate equations (32a) and (32b), or equivalently to satisfy the combined equation (33). The fact that each of these equations is invariant to a permutation of $\{a, b, c\}$ or of $\{X, Y, Z\}$ is reflected on a similar invariance for the set of particular solutions in equations (37) or (38). This means that if a permutation of $\{a, b, c\}$ or a permutation of $\{X, Y, Z\}$ is applied to any particular solution, then it remains a particular solution (albeit possibly a different one).

In passing, we note that the number of particular solutions of each of the individual equations (32a) and $(32 b)$ is given by

$$
n=(1)^{2} *(7)^{7}=823543, \quad i=1,2,
$$

i.e., it is about 17.65 times the number of solutions of the system of equations (38). The following equation (41) displays all 823543 particular solutions of the equation (32a) as a disjunction of possible contributions of atoms $T_{i}(1 \leq i \leq 7)$.

$$
\left[\begin{array}{l}
X \\
Y \\
Z
\end{array}\right]=\mathrm{V}_{\mathrm{i}=1}^{7}
$$

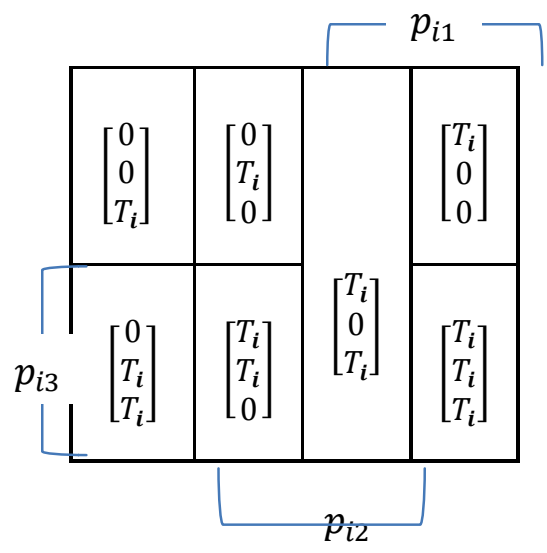


Similarly, The following equation (42) displays all 823543 particular solutions of the system (32b) as a disjunction of possible contributions of atoms $T_{i}(1 \leq i \leq 7)$.

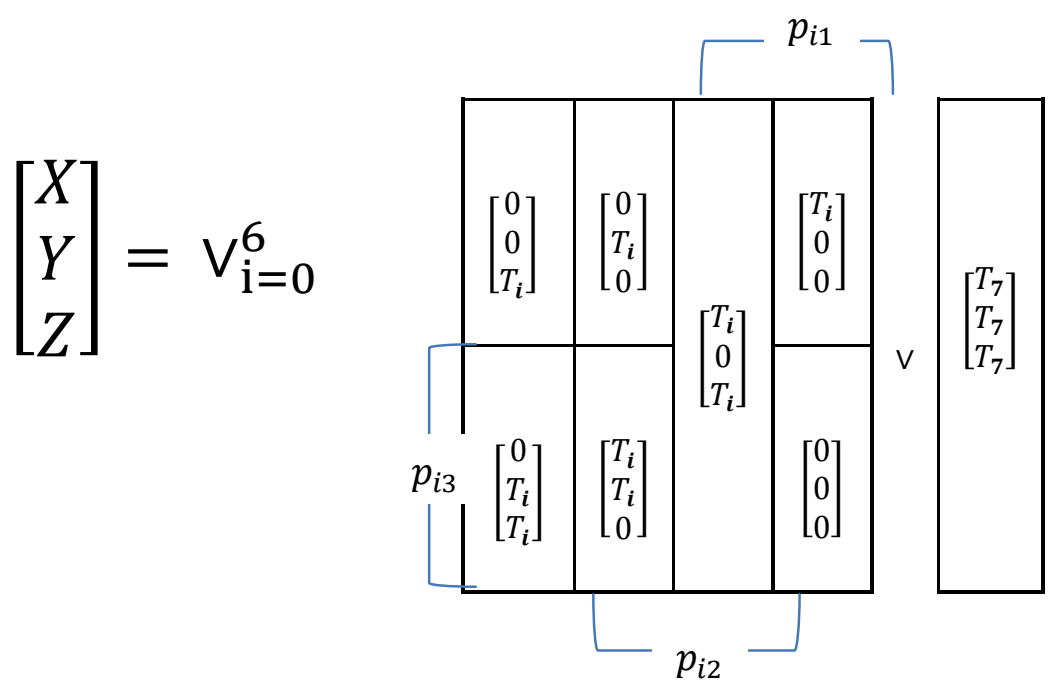

The intersection of the set of solutions in Equation (41) with the set of solutions in Equation (42) is the set of solutions in Equation (38). In fact, the set of solutions in Equation (38) do not contain a contribution of $T_{0}$ and must contain a contribution (to each variable) of $T_{7}$. This means that this set is the intersection of the $(6)^{7}=117649$ solutions in Fig. 6 that has a contribution of $T_{7}$ of the form $\left[\begin{array}{llll}T_{7} & T_{7} & T_{7}\end{array}\right]^{T}$, with the $(6)^{7}$ solutions in Equation (42) that have zero contribution of $T_{0}$ of the form $\left[\begin{array}{lll}0 & 0 & 0\end{array}\right]^{T}$. When we add the restriction that solutions of the form $\left[\begin{array}{lll}T_{\mathrm{i}} & T_{\mathrm{i}} & T_{\mathrm{i}}\end{array}\right]$ for $1 \leq i \leq 6$ in Equation (41) should be excluded, we retain only the 46656 solutions in Equation (38). Likewise when we add the restriction that no contribution of $T_{\mathrm{i}}$ for $(1 \leq i \leq 6)$ in Equation (42) should be of the form $\left[\begin{array}{lll}0 & 0 & 0\end{array}\right]^{T}$, we again retain only the 46656 solutions in Equation (38). Since the two sets of solutions in Equation (41) and (42) are now restricted to match the set in Equation (38), their intersection is also equal to this set.

Before closing this example, we include its general subsumptive solution, obtained in the most compact form via the don't-care method $[13,15]$. This solution includes the consistency condition $(0=0)$ together with the subsumptive relations

$$
\begin{aligned}
& a b c \leq Z \leq(a \vee b \vee c) \\
& a b c \leq Y \leq(a \vee b \vee c) \\
& a b c \vee(a \vee b \vee c) \bar{Y} \bar{Z} \leq X \leq a b c \vee(a \vee b \vee c)(\bar{Y} \vee \bar{Z})
\end{aligned}
$$

which might be rewritten in terms of atoms $T_{\mathrm{i}}(0 \leq i \leq 7)$ as follows

$$
\begin{aligned}
& T_{7} \leq Z \leq\left(\mathrm{V}_{i=1}^{7} T_{i}\right) \\
& T_{7} \leq Y \leq\left(\mathrm{V}_{i=1}^{7} T_{i}\right) \\
& T_{7} \vee\left(\vee_{i=1}^{6} T_{i}\right) \bar{Y} \bar{Z} \leq X \leq T_{7} \vee\left(\vee_{i=1}^{6} T_{i}\right)(\bar{Y} \vee \bar{Z}) .
\end{aligned}
$$

A straightforward (albeit lengthy) analysis shows that (44) is in exact agreement with Equation (38). 
Table 1. Samples of the particular solutions of Example 1

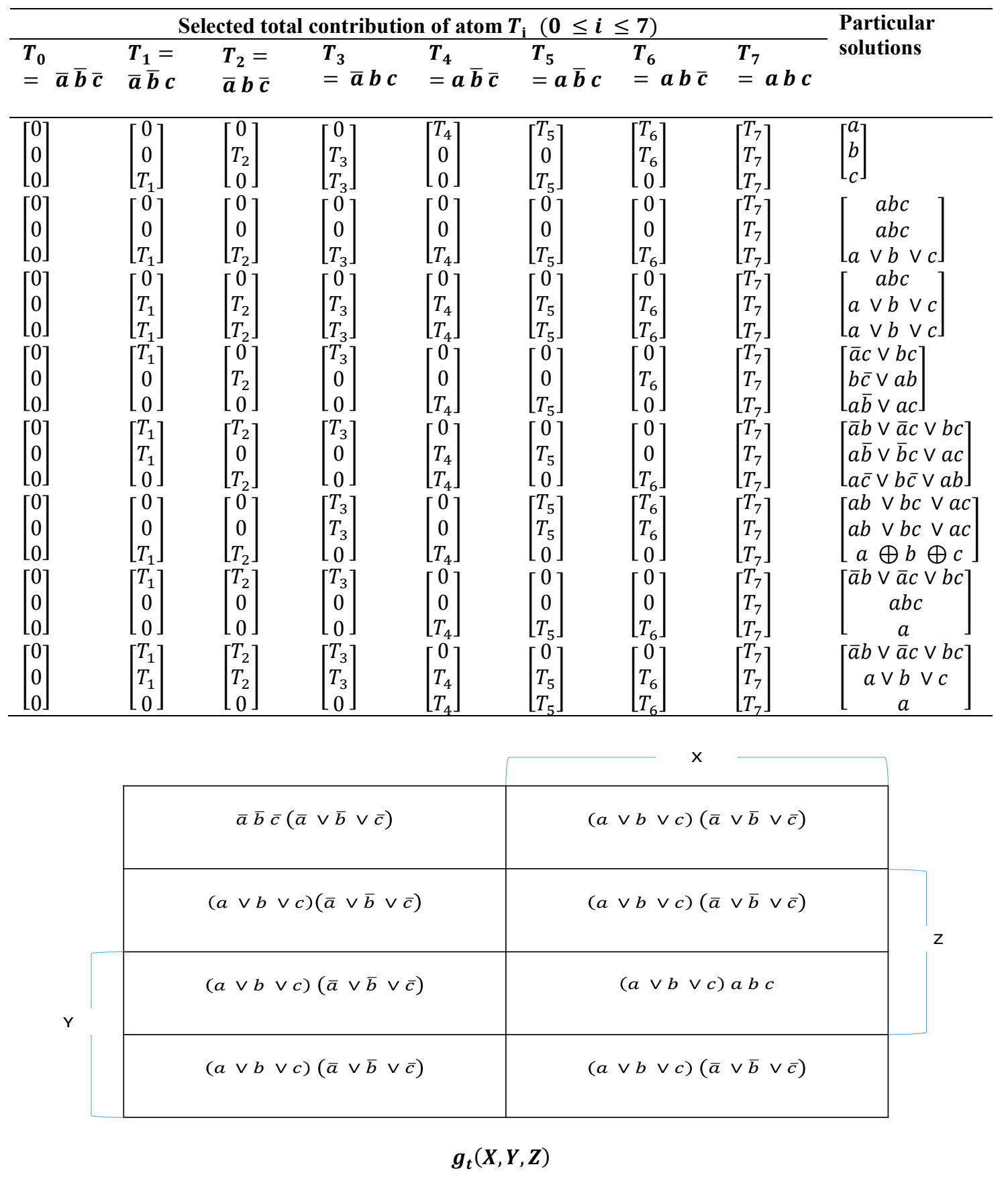

Fig. 8. The natural map for $g_{t}(X, Y, Z)$ in (33) 


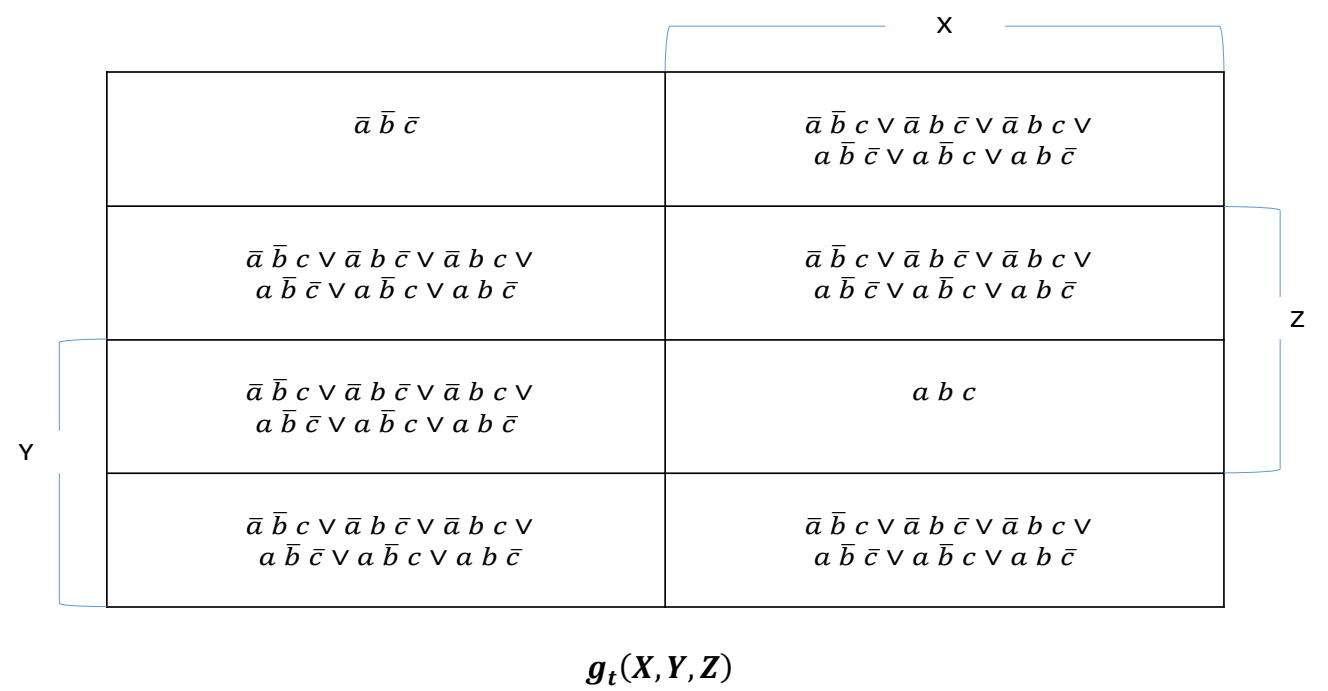

Fig. 9. The map in Fig. 8 with its entries given as minterm expansions (disjunctions of the atoms of the underlying Boolean algebra)

\begin{tabular}{|c|c|}
\hline \multicolumn{1}{|c|}{$T_{0}(1)$} & $\mathrm{x}$ \\
\hline$\bigvee_{i=\mathbf{1}}^{6} T_{i}\left(\overline{p_{i 1}} \overline{p_{i 2}} \overline{p_{i 3}}\right)$ & $\bigvee_{i=\mathbf{1}}^{6} T_{i}\left(p_{i 1} \overline{p_{i 2}}\right)$ \\
\hline$\bigvee_{i=\mathbf{1}}^{6} T_{i}\left(\overline{p_{i 1}} \overline{p_{i 2}} p_{i 3}\right)$ & $\bigvee_{i=\mathbf{1}}^{6} T_{i}\left(p_{i 1} p_{i 2}\right)$ \\
\hline$\bigvee_{i=\mathbf{1}}^{6} T_{i}\left(\overline{p_{i 1}} p_{i 2} \overline{p_{i 3}}\right)$ & $T_{7}(1)$ \\
\hline \\
\hline
\end{tabular}

Fig. 10. The auxiliary function for Example 3

\section{Conclusions}

In this paper we introduced a novel method for listing all the particular solutions of a system of 'big' Boolean equations. The method needs a very compact space even when the number of particular solutions is very large. Such a compact listing is helpful in selecting certain particular solutions of desirable features. The reason why such a compact listing is possible was the expression of particular solutions as the sum (ORing) of certain contributions of asserted atoms of the underlying Boolean algebra, which appear in the discriminants of the pertinent Boolean function. 
The paper reiterates a thesis endorsed by Brown $[1,2]$ that the use of 'big' Boolean algebras is unavoidable. This unavoidability is definitely manifested in prominent engineering applications. For example, the paper considers the case of digital design in which it is desirable to solve for some output variables in terms of some input variables. Though both input and output variables are actually switching variables (two-valued Boolean variables), the case is handed by treating the input variables as generators of a 'big' Boolean algebra, and handling the output variables as elements belonging to the carrier of this Boolean algebra.

The paper strives to make the solution of 'big' Boolean equations easily accessible to a wider audience of practitioners. Therefore, it demonstrates its method via a variety of examples that not only illustrate its mathematical steps but also unravel many of the associated subtle, intricate, or occasionally unclear concepts including the reduction of a system of equations to a single equation, the relation between the solution of this single equation and the solutions of the original equations, the complementation of a Boolean function to obtain its inverse, the collapse of a 'big' Boolean algebra to one of its sub algebras due to the consistency condition, the incremental solution of an equation in terms of a related one, and the exact agreement between solutions obtained via parametric and subsumptive methods.

\section{Competing Interests}

Authors have declared that no competing interests exist.

\section{References}

[1] Brown FM. Boolean reasoning: The logic of Boolean equations, Kluwer Academic Publishers, Boston, USA; 1990.

[2] Brown FM. Boolean reasoning: The logic of Boolean equations, $2^{\text {nd }}$ Ed., Dover Publications, Mineola, NY, USA; 2003.

[3] Rudeanu S. Boolean functions and equations. North-Holland Publishing Company \& American Elsevier, Amsterdam, the Netherlands; 1974.

[4] Rudeanu S. Lattice functions and equations. Springer Verlag, London, UK; 2001.

[5] Leont'ev VK, Tonoyan GP. On systems of Boolean equations. Computational Mathematics and Mathematical Physics. 2013;53(5):632-639.

[6] Levchenkov VS. Boolean equations with many unknowns. Computational Mathematics and Modeling. 2000;11(2):143-153.

[7] Levchenkov VS. Solution of equations in Boolean algebra. Computational Mathematics and Modeling. 2000;11(2):154-163.

[8] Rudeanu S. Algebraic methods versus map methods of solving Boolean equations. International Journal of Computer Mathematics. 2003;80 (7):815-817.

[9] Rushdi AM. Using variable-entered Karnaugh maps to solve Boolean equations. International Journal of Computer Mathematics. 2001;78(1):23-38.

[10] Rushdi AM. Efficient solution of Boolean equations using variable-entered Karnaugh maps. Journal of King Abdulaziz University: Engineering Sciences. 2004;15(1):105-121.

[11] Rushdi AM. A comparison of algebraic and map methods for solving general Boolean equations. Journal of Qassim University: Engineering and Computer Sciences. 2012;4(2):1-32. 
[12] Rushdi AM, Amashah MH. Purely-algebraic versus VEKM methods for solving big Boolean equations. Journal of King Abdulaziz University: Engineering Sciences. 2012;23(2):75-85.

[13] Rushdi AMA, Albarakati HM. Prominent classes of the most general subsumptive solutions of Boolean equations. Information Sciences. 2014;281:53-65.

[14] Tucker JH, Tapia MA. Using Karnaugh maps to solve Boolean equations by successive elimination. Proceedings of IEEE Southeastcon 92. Birmingham, AL, USA. 1992;2:589-592.

[15] Rushdi AM. Improved variable-entered Karnaugh procedures. Computer and Electrical Engineering. $1987 ; 13(1): 41-52$.

[16] Rushdi AM, Al-Yahya HA. A Boolean minimization procedure using the variable-entered Karnaugh map and the generalized consensus concept. International Journal of Electronics. 2000;87(7):769-794.

[17] Rushdi AM, Al-Yahya HA. Further improved variable-entered Karnaugh map procedures for obtaining the irredundant forms of an incompletely-specified switching function. Journal of King Abdulaziz University: Engineering Sciences. 2001;13(1):111-152.

[18] Rushdi AM, Amashah MH. Parametric general solutions of Boolean equations via variable-entered Karnaugh maps. Journal of Qassim University: Engineering and Computer Sciences. 2010;3(1):59-71.

[19] Rushdi AM, Amashah MH. Using variable-entered Karnaugh maps to produce compact parametric general solutions of Boolean equations. International Journal of Computer Mathematics. 2011;88(15): 3136-3149.

[20] Banković D. Some remarks on number of parameters of the solutions of Boolean equations. Discrete Mathematics. 1990;79(3):229-234.

[21] Brown FM. Reduced solutions of Boolean equations. IEEE Transactions on Computers. 1970;C-19: 976-981.

[22] Brown FM. Segmental solutions of Boolean equations. Discrete Applied Mathematics. 1982;4:87-96.

[23] Deschamps JP. Parametric solutions of Boolean equations. Discrete Mathematics. 1972;3(4):333-342.

(C) 2017 Rushdi and Ahmad; This is an Open Access article distributed under the terms of the Creative Commons Attribution License (http://creativecommons.org/licenses/by/4.0), which permits unrestricted use, distribution, and reproduction in any medium, provided the original work is properly cited.

Peer-review history

The peer review history for this paper can be accessed here (Please copy paste the total link in your

browser address bar)

http://sciencedomain.org/review-history/19556 